\title{
Stability assessment and improvement of a Lactobacillus plantarum mutant with low post-fermentation acidification characteristics
}

\author{
L.-O. Chuah $(\mathbb{0}$ and Y. Mao* (1) \\ DuPont Nutrition and Biosciences, Shanghai 200335, P. R. China
}

\section{ABSTRACT}

Intracellular $\mathrm{pH}$ homeostasis through the extrusion of a proton by $\mathrm{F}_{0} \mathrm{~F}_{1}$-ATPase is one of the key mechanisms used by lactic acid bacteria in response to acid stress, and also influences their post-fermentation acidification. In this study, the genotypic and phenotypic stability of a low post-fermentation acidification (LPA) mutant (designated as DGCC12411 ${ }^{\mathrm{m}}$ ) of Lactobacillus plantarum DGCC12411 was assessed. Compared with its mother strain, the $\mathrm{pH}$ of $\mathrm{DGCC} 12411^{\mathrm{m}}$ in De Man, Rogosa, and Sharpe (MRS) broth after 48-h cultivation was $0.35 \mathrm{pH}$ units higher. Incorporation of DGCC $12411^{\mathrm{m}}$ in yogurt stored at ambient temperature (ambient yogurt) showed a reduced post-fermentation acidification during storage at $25^{\circ} \mathrm{C}$ for $120 \mathrm{~d}$. Wholegenome sequencing analysis showed a SNP mutation (GGT > GAT at positions 505 to 507) in DGCC12411 ${ }^{\mathrm{m}}$, which resulted in the substitution of a highly conserved glycine residue by aspartic acid at the Walker A motif of the $\mathrm{F}_{0} \mathrm{~F}_{1}$-ATPase $\alpha$-subunit. However, degeneration of the LPA phenotype was observed after 5 passages of DGCC $12411^{\mathrm{m}}$ in MRS broth. Analysis of DNA sequencing on both the whole population and the isolates showed that a back mutation occurred at the SNP site (GAT changed back to GGT) over the passaging, and the reversion gradually increased from a ratio of $10.8 \%$ at P5 to $60.0 \%$ at P10. We also found that the LPA phenotype stability of DGCC $12411^{\mathrm{m}}$ was improved by supplementing $0.1 \mathrm{M}$ potassium phosphate buffer to the growth medium as well as by reducing the inoculation rate of $\mathrm{DGCC} 12411^{\mathrm{m}}$ to $2 \%$ (vol/vol). Such LPA Lactobacillus strains have potential for use as starter cultures in fermented foods with less change in acidity during shelf-life storage.

Key words: Lactobacillus plantarum, $\mathrm{F}_{0} \mathrm{~F}_{1}$-ATPase, low post-fermentation acidification, acid-sensitive mutant

Received January 30, 2020.

Accepted April 22, 2020.

*Corresponding author: Yuejian.Mao@dupont.com

\section{INTRODUCTION}

Most of the fermented foods consumed around the world are produced by lactic acid bacteria (LAB), which utilize carbohydrates from different raw materials to produce lactic acid as the main final product of their metabolism (Piard and Desmazeaud, 1991; Tamang et al., 2016). Lactobacillus is one of the most important LAB taxa present in fermented foods such as dairy, meats, and vegetable products (Yang et al., 2010; $\mathrm{Yu}$ et al., 2015). Owing to its resistance to low $\mathrm{pH}$ and ability to utilize a wide range of carbohydrate sources, Lactobacillus plantarum has been used as starter culture in various food fermentations such as a nondairy fermented beverage from a blend of cassava and rice (Freire et al., 2017), gowé (a fermented beverage made from malted and nonmalted sorghum flour; de J. C. Munanga et al., 2016), and fermented raw-dried meat sausages (Nedelcheva et al., 2010). It contributes significantly to the organoleptic properties, such as flavor and texture, of fermented foods (Bernardeau et al., 2006). However, strong post-fermentation acidification by $L$. plantarum limits its broader industrial application in fermented foods, such as dairy products, for which consumers are increasingly showing a preference for a relatively mild taste. Studies focused on starter cultures' post-fermentation acidification improvement have been reported (Möller et al., 2007; Gomez et al., 2017). Therefore, to understand the science in controlling post-fermentation acidification and developing methods to generate low post-fermentation acidification (LPA) variants are valuable to its wider industrial application. Low post-fermentation acidification is generally defined as $\mathrm{pH}$ value declining less than or equal to $0.3 \mathrm{pH}$ units from $\mathrm{pH} 4.5$ in an extended fermentation period of $24 \mathrm{~h}$, according to industrial practice (Mao, 2019).

In addition to using lactose-negative Lactobacillus strains to control the post-fermentation acidification in yogurt (Chen et al., 2018), selection of acid-sensitive mutants, which are unable to continue fermentation below a certain $\mathrm{pH}$, is another promising approach to improve control of post-fermentation acidification. 
$\mathrm{F}_{0} \mathrm{~F}_{1}$-ATPase is a key enzyme in maintaining intracellular $\mathrm{pH}$ through extrusion of protons out of the cells by hydrolysis of ATP (Cotter and Hill, 2003). Several studies have reported that the basal activity of the $\mathrm{F}_{0} \mathrm{~F}_{1^{-}}$ ATPase was lower in LAB strains that were more sensitive to low pH (Nannen and Hutkins, 1991; Yamamoto et al., 1996; Ongol et al., 2007). Kanner and Gutnick (1972) demonstrated a negative correlation between ATPase activity and neomycin resistance in Escherichia coli. Reduced $\mathrm{F}_{0} \mathrm{~F}_{1}$-ATPase activity resulted in reduction of aminoglycoside antibiotics uptake, thus increasing neomycin resistance (Kanner and Gutnick, 1972). By using neomycin as selection pressure, spontaneous acid-sensitive variants have been successfully derived from Lactococcus lactis ssp. lactis (Yokota et al., 1995), Pediococcus acidilactici (Kurdi et al., 2009), Lactobacillus helveticus (Yamamoto et al., 1996), Lactobacillus delbrueckii ssp. bulgaricus (Ongol et al., 2007; Liu et al., 2017), and Lactobacillus plantarum (Jaichumjai et al., 2010; Jia et al., 2013).

Because $\mathrm{F}_{0} \mathrm{~F}_{1}$-ATPase is extremely conserved during evolution and is critical to bacterial physiological activities (Koumandou and Kossida, 2014), a mutation in this gene that reduces ATPase activity and increases acid sensitivity may cause competitive disadvantages in growth media or food matrices without neomycin pressure. Subsequently, this may influence the mutant's stability and performance in food fermentation. However, the stability of $\mathrm{F}_{0} \mathrm{~F}_{1}$-ATPase mutation in LPA LAB mutants has never been assessed. In this study, an acid-sensitive $L$. plantarum variant with an SNP mutation on its $\mathrm{F}_{0} \mathrm{~F}_{1}$-ATPase $\alpha$-subunit was generated. To understand the performance of DGCC $12411^{\mathrm{m}}$ in industrial application, we tested its post-fermentation acidification and cell count during shelf-life storage in a yogurt stored at ambient temperature (hereafter termed "ambient yogurt"). We selected this application model because ambient yogurt is the largest fermented dairy product category in China, with continued high sales growth. In addition, genotypic and phenotypic stability of this variant were evaluated. Methods to improve its stability during propagation were also developed.

\section{MATERIALS AND METHODS}

\section{Strain and Growth Conditions}

The strain L. plantarum DGCC12411 was obtained from the DuPont Global Culture Collection (Niebüll, Germany); this strain was originally isolated from a traditional fermented vegetable product. This strain was deposited in Deutsche Sammlung von Mikroorganismen und Zellkulturen GmbH (Braunschweig, Germany) under the accession number DSM32493. The strain was stored in $15 \%$ ( $\mathrm{vol} / \mathrm{vol}$ ) glycerol at $-80^{\circ} \mathrm{C}$ until use. The working cultures of DGCC12411 and its mutant strains were prepared by inoculating $1 \%$ (vol/ vol) of the glycerol stock of each culture into fresh De Man, Rogosa, and Sharpe (MRS) broth (Oxoid, Basingstoke, UK) and incubated aerobically, under ambient gas conditions, at $30^{\circ} \mathrm{C}$ for $24 \mathrm{~h}$. Similar aerobic cultivation in MRS medium was used throughout the experiment, unless mentioned otherwise.

\section{Generated LPA Mutants}

The acid-sensitive mutants were generated as previously described (Ongol et al., 2007), with minor modification. The DGCC12411 was revived by dissolving 100 $\mathrm{mg}$ of freeze-dried culture in $10 \mathrm{~mL}$ of sterile phosphate buffer (3M Co., Maplewood, MN), serially diluted, and plated at the appropriate dilution on MRS agar. The MRS agar plates were incubated at $30^{\circ} \mathrm{C}$ for $48 \mathrm{~h}$. A single colony was picked and transferred into $10 \mathrm{~mL}$ of MRS broth and incubated at $30^{\circ} \mathrm{C}$ overnight until midexponential phase [optical density $(\mathbf{O D})_{595 \mathrm{~nm}}=0.8$ to $0.9]$. The OD reading was measured using Tecan Infiniti PRO 200 spectrophotometer (Thermo Fisher Scientific, Waltham, MA). To derive acid-sensitive mutants from DGCC12411, $200 \mu \mathrm{L}[2 \%$ (vol/vol)] of the overnight culture was transferred to $10 \mathrm{~mL}$ of half-strength $(26 \mathrm{~g} / \mathrm{L})$ MRS broth containing $700 \mu \mathrm{g} / \mathrm{mL}$ neomycin sulfate (CAS 1405-10-3; Rebio Technologies Ltd., Cranleigh, $\mathrm{UK}$; purity $\geq 98 \%$ ) and incubated at $30^{\circ} \mathrm{C}$ for 48 to 72 $\mathrm{h}$ until mid-exponential phase $\left(\mathrm{OD}_{595 \mathrm{~nm}}\right.$ approximately $0.5)$. Subsequently, the culture was diluted in a sterile phosphate buffer (3M Co.) and appropriate dilutions plated on the half-strength MRS agar containing 700 $\mu \mathrm{g} / \mathrm{mL}$ neomycin sulfate. The agar plates were then incubated at $30^{\circ} \mathrm{C}$ for 3 to $5 \mathrm{~d}$, until visible colonies were observed. Two thousand single-colony isolates were randomly selected and grown in 96-well microtiter plates (Nunc, Roskilde, Denmark) containing $200 \mu \mathrm{L}$ of MRS broth ( $\mathrm{pH} 6.3$ ) per well and incubated at $30^{\circ} \mathrm{C}$ for $48 \mathrm{~h}$. After incubation, 1\% (vol/vol) culture from each well of the microtiter plates was inoculated into 2 new microtiter plates containing $100 \mu \mathrm{L} /$ well of fresh medium: one containing MRS broth at $\mathrm{pH} 6.3$, and the other containing MRS broth adjusted to $\mathrm{pH}$ 4.5. The microtiter plates were incubated at $30^{\circ} \mathrm{C}$ for $72 \mathrm{~h}$. Variants that grew well, as indicated by turbidity change at $\mathrm{pH} 6.3\left(\mathrm{OD}_{595 \mathrm{~nm}} \geq 1.0\right.$ at $72 \mathrm{~h}$ after subtracted with the blank) but not at $\mathrm{pH} 4.5\left(\mathrm{OD}_{595 \mathrm{~nm}} \leq 0.3\right.$ at $72 \mathrm{~h}$ after subtraction of the blank) were recognized as potential acid-sensitive mutants of DGCC12411.

The post-fermentation acidification of 8 selected variants was further validated by assessing their acidification in MRS. In brief, both DGCC12411 and the 
potential variants were grown in $10 \mathrm{~mL}$ of MRS broth at $30^{\circ} \mathrm{C}$ for $24 \mathrm{~h}$. The cultures were adjusted to $\mathrm{OD}_{595 \mathrm{~nm}}$ 0.5 and inoculated at $2 \%(\mathrm{vol} / \mathrm{vol})$ to $100 \mathrm{~mL}$ of fresh MRS broth. The culture was then incubated at $30^{\circ} \mathrm{C}$ in a water bath, and the acidification activity of each strain was monitored by iCINAC (AMS Alliance, Rome, Italy) every $3 \mathrm{~min}$ for $60 \mathrm{~h}$. One mutant (designated as DGCC $12411^{\mathrm{m}}$ ) showing the highest stable final $\mathrm{pH}$ was selected for further study.

\section{Post-Fermentation Acidification and Cell Count Assessment in Ambient Yogurt}

A commercial ambient yogurt product is a fermented milk product that has been subjected to pasteurization after the end of fermentation. The pasteurization step inactivates the starter cultures used for fermentation, and this product contains no living cells. In this study, DGCC12411 and its mutant strain were respectively supplemented to commercially available ambient yogurt as adjunct cultures. Approximately $1.0 \times 10^{7} \mathrm{cfu} / \mathrm{mL}$ of DGCC12411 or DGCC12411 ${ }^{\mathrm{m}}$ were inoculated into a commercial pasteurized ambient yogurt (Momchilovtsi, Bright Dairy, Shanghai, China) and stored at $25^{\circ} \mathrm{C}$ for $120 \mathrm{~d}$. The $\mathrm{pH}$ and cell count were measured at d 0,30 , 60,90 , and 120. For cell count enumeration, the yogurt samples inoculated with DGCC12411 or its mutant strain were mixed well using a vortexer, and $11 \mathrm{~g}$ of the homogeneous sample was diluted in $99 \mathrm{~mL}$ of sterile phosphate buffer (3M Co.). Subsequently, 10-fold serial dilution was performed by using sterile phosphate buffer (3M Co.), and $100 \mu \mathrm{L}$ of appropriate dilutions were spread-plated onto the MRS agar. The MRS agar plates were then incubated at $30^{\circ} \mathrm{C}$ for $48 \mathrm{~h}$, followed by colony enumeration. Three individual replicates were performed for each treatment for $\mathrm{pH}$ and cell count measurements.

\section{ATPase Gene PCR and Sequence Analysis}

Based on the aligned $\mathrm{F}_{0} \mathrm{~F}_{1}$-ATPase gene sequences of DGCC12411 and DGCC12411 ${ }^{\mathrm{m}}$, primers flanking the mutation site (ATPase-F: 5'-TCAACCAGTTGACGGTTCAG-3', and ATPase-R: 5'-TTTGGTTCAAAATGGCATCA-3') were designed. The 40$\mu \mathrm{L}$ PCR reaction mixture contained $0.5 \mu \mathrm{L}$ of overnight cultures in $1 \times$ buffer, $2.5 \mathrm{mM} \mathrm{MgCl} 2$, dNTPs and 0.04 U Taq DNA polymerase (each $0.5 \mathrm{~m} M$; Promega, Madison, WI), and $0.25 \mu M$ primers (MajorBio, Shanghai, China). The PCR reaction was performed using a Veriti 96 Well Thermal Cycler (Applied Biosystems, Waltham, MA) using the following amplification protocol: initial denaturation at $95^{\circ} \mathrm{C}$ for $5 \mathrm{~min} ; 30$ cycles of $95^{\circ} \mathrm{C}$ for $45 \mathrm{~s}, 52^{\circ} \mathrm{C}$ for $45 \mathrm{~s}$, and $72^{\circ} \mathrm{C}$ for $30 \mathrm{~s}$; and a final extension at $72^{\circ} \mathrm{C}$ for $5 \mathrm{~min}$. The PCR amplicons were loaded onto a 1.5\% (wt/vol) agar gel pre-stained with SyBr Safe DNA dye (Invitrogen, Carlsbad, CA) for direct visualization after electrophoresis for $60 \mathrm{~min}$ at $100 \mathrm{~V}$. Molecular ladders of $100 \mathrm{bp}$ (New England Biolabs, Ipswich, MA) were used to estimate the size of the PCR amplicons (approximately $224 \mathrm{bp}$ ). Subsequently, PCR amplicons were subjected to gel purification, and Sanger sequencing analysis was performed by using ATPase-F primer (MajorBio).

The DNA sequencing results were processed using Seq Scanner 2 software (https://www.thermofisher .com/us/en/home/life-science/sequencing/sanger -sequencing/sanger-dna-sequencing/sanger-sequencing -data-analysis.html). We used BLASTN (Morgulis et al., 2008; Zhang et al., 2000) to confirm the identity of the gene sequences by comparison with type strains available at the National Center for Biotechnology Information (NCBI, Bethesda, MD). The ATPase gene sequences of DGCC12411 and DGCC12411 ${ }^{\mathrm{m}}$ were aligned and compared by ClustalW (https://embnet .vital-it.ch/software/ClustalW.html), using L. plantarum WCFS1 (YP_004890068.1) as reference.

\section{Stability Assessment of DGCC12411 ${ }^{m}$ Strain}

Stability of the LPA phenotype and the $\mathrm{F}_{0} \mathrm{~F}_{1}$-ATPase SNP mutation of DGCC $12411^{\mathrm{m}}$ over 10 passages were assessed. With the $10 \%$ ( vol/vol) inoculation rate, we estimated that 3.3 generations occurred in each passage, a total of 33 generations throughout 10 passages. The $\mathrm{pH}$ after 48 -h cultivation $\left(\mathbf{p H}_{48 \mathrm{~h}}\right)$ was used as the post-fermentation acidification indicator. Three colonies of DGCC $12411^{\mathrm{m}}$ were randomly chosen and inoculated from the MRS agar plate into $10 \mathrm{~mL}$ tubes of MRS broth, respectively, and incubated at $30^{\circ} \mathrm{C}$ for $24 \mathrm{~h}$. Then, $1 \mathrm{~mL}$ of fresh culture from each tube was inoculated into a new tube containing $9 \mathrm{~mL}$ of fresh MRS medium and incubated at $30^{\circ} \mathrm{C}$ for $24 \mathrm{~h}$. After 24-h incubation, these cultures were designated as passage $1(\mathbf{P 1})$ and subsequently used as the inoculum for passage 2 (P2). The initial culture used to inoculate $\mathrm{P} 1$ was further incubated at $30^{\circ} \mathrm{C}$ for another $24 \mathrm{~h}$ for the $\mathrm{pH}$ measurement (Mettler Toledo $\mathrm{pH}$ meter, Greifensee, Switzerland) after $48 \mathrm{~h}$ of incubation. The same process was repeated for another 9 consecutive passages (P2 to P10). The mother strain DGCC12411 was used as a control. The $\mathrm{F}_{0} \mathrm{~F}_{1}$-ATPase $\alpha$-subunit gene of each passage was sequenced, and $100 \mu \mathrm{L}$ of the 24 -h broth culture of each passage was serially diluted and plated on MRS plates. At each passage, 10 colonies per sample were selected for further post-fermentation acidification and ATPase gene analysis. 


\section{Effects of Inoculum Size and Potassium Phosphate Buffer on Phenotypic Stability}

Effects of inoculum size on phenotypic stability were measured by inoculating 2 or $10 \%$ (vol/vol) DGC$\mathrm{C} 12411^{\mathrm{m}}$ into MRS broth for 10 passages, using the method previously described. With the $2 \%$ (vol/vol) inoculation rate, we estimated that 5.6 generations occurred in each passage, hence a total of 56 generations throughout 10 passages. We used MRS supplemented with neomycin sulfate $(600 \mu \mathrm{g} / \mathrm{mL})$ as a positive control. The effect of potassium phosphate buffer was assessed by comparing phenotype stability between the untreated control and MRS medium supplemented with 0.05 or $0.10 \mathrm{M}$ potassium phosphate buffer (7.214 $\mathrm{g}$ of sodium phosphate dibasic heptahydrate and 10.086 $\mathrm{g}$ of sodium phosphate monobasic monohydrate in $1 \mathrm{~L}$ of MRS medium; $\mathrm{pH}$ adjusted to 6.3). Three individual replicates were performed for each test.

\section{Statistical Analysis}

Data were statistically analyzed with Minitab software (version 18; Minitab Inc., State College, PA). One-way ANOVA (Tukey test) was used to study the significant differences between means, with significant difference at $P<0.05$ or $P<0.01$.

\section{Sequence Deposition}

The $\mathrm{F}_{0} \mathrm{~F}_{1}$-ATPase gene nucleotide sequences of DGCC12411 and DGCC12411 ${ }^{\mathrm{m}}$ were deposited in GenBank under accession numbers MN886309 and MN886310.

\section{RESULTS AND DISCUSSION}

\section{LPA L. plantarum Variant and the Putative Causal Mutation}

Lactobacillus plantarum DGCC12411 is of high industrial interest, as it is an exopolysaccharide-producing bacterium that contributes to high thickness and ropiness texture for fermented food. However, controlling its post-fermentation acidification during food fermentation is challenging. To reduce its postfermentation acidification, a spontaneous mutation and a selective screening method were adopted to generate natural LPA variants. After screening of 2,000 isolates, 8 variants that could not grow in acidified MRS broth ( $\mathrm{pH} 4.5)$ were obtained. By monitoring their acidification in normal MRS ( $\mathrm{pH}$ 6.3), we found that 7 variants were slow in acidification but stabilized at the same $\mathrm{pH}$ as the mother strain after 60 -h fermentation (data

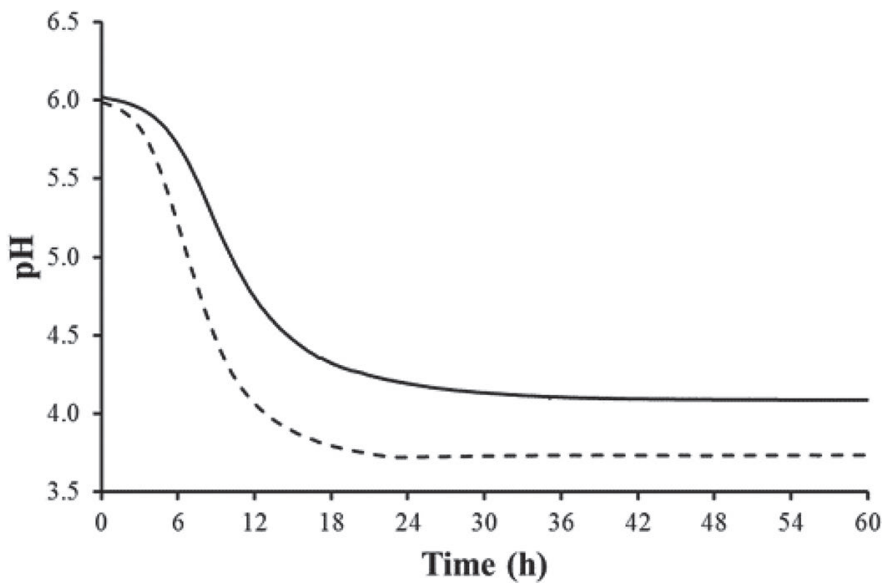

Figure 1. Acidification curves of Lactobacillus plantarum DGCC12411 (dashed line) and its acid-sensitive mutant strain (DGCC12411 ${ }^{\mathrm{m}}$; solid line) in De Man, Rogosa, and Sharpe broth. Each treatment has duplicates.

not shown). Only DGCC $12411^{\mathrm{m}}$ with $\mathrm{pH} 4.21$ at 24 $\mathrm{h}$ was selected as an LPA variant. At extended fermentation to $60 \mathrm{~h}, \mathrm{DGCC} 12411^{\mathrm{m}}$ stopped at $\mathrm{pH} 4.09$, which is $0.35 \mathrm{pH}$ unit higher than the mother strain DGCC12411 (Figure 1). The pH of both DGCC12411 and DGCC $12411^{\mathrm{m}}$ remained stable for 36 to $60 \mathrm{~h}$ of culture; thus we selected the $\mathrm{pH}$ at $48 \mathrm{~h}$, a central point, as an end $\mathrm{pH}$ to compare the post-fermentation acidification of DGCC12411 and its variants in further tests.

The industry is looking for cultures for use in ambient yogurt products that possess LPA effects during ambient-temperature storage. However, we also noticed that DGCC $12411^{\mathrm{m}}$ was slower than its mother strain in fermentation (Figure 1). This is a disadvantage for starter cultures in applications that require fast fermentation but an advantage for adjunct cultures in foods, especially those stored at ambient temperature, when it is desirable to minimize the strain's acidification activity during shelf-life for better product stability. In this study, after inoculation of $1 \times 10^{7} \mathrm{cfu} / \mathrm{mL} \mathrm{DGCC} 12411^{\mathrm{m}}$ or DGCC12411 into the ambient yogurt and storage at $25^{\circ} \mathrm{C}$, a significant drop in $\mathrm{pH}$ mainly occurred during the first $2 \mathrm{mo}$; then the $\mathrm{pH}$ of the yogurt became stable for the yogurts inoculated with either strain. Consistent with the acidification results obtained using MRS, DGCC12411 ${ }^{\mathrm{m}}$ also showed better $(P<0.01)$ post-fermentation acidification control in the ambient yogurt, with a $\Delta \mathrm{pH}$ of $0.2 \mathrm{pH}$ unit higher than the parent at d 30 and 0.12 at d 120 (Figure 2A). Changes in the taste of the yogurt inoculated with DGCC12411 ${ }^{\mathrm{m}}$ appeared more acceptable than with DGCC12411 due to its lower acidity (data not shown). The cell count of DGCC $12411^{\mathrm{m}}$ in ambient yogurt remained at or slightly higher than $1 \times 10^{7} \mathrm{cfu} / \mathrm{mL}$ for up to $60 \mathrm{~d}$, and only 1 

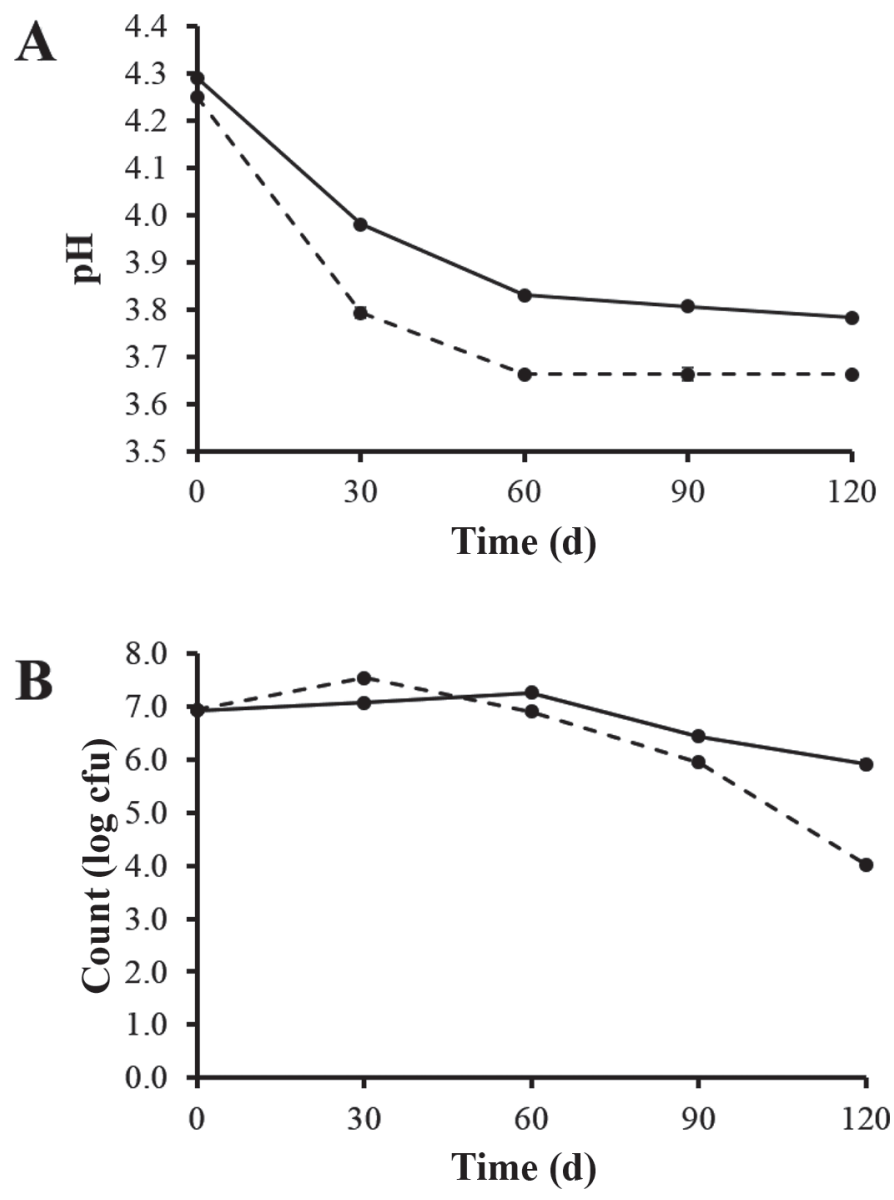

Figure 2. (A) $\mathrm{pH}$ and (B) cell count of Lactobacillus plantarum DGCC12411 (dashed line) and its acid-sensitive mutant DGCC12411 (solid line) in the model of yogurt at ambient temperature during shelf-life storage $\left(25^{\circ} \mathrm{C}\right.$ for $\left.120 \mathrm{~d}\right)$. Error bars represent SD of 3 replicates.

log reduction in cell count was observed on d 120. Our results ruled out the possibility that the improvement in post-acidification control was due to reduction in cell count during shelf-life.

To identify possible mutations responsible for the LPA phenotype, the whole genome of DGCC $12411^{\mathrm{m}}$ was sequenced and compared with its mother strain (data not shown). An SNP mutation (GGT > GAT at positions 505 to 507) in the $\mathrm{F}_{0} \mathrm{~F}_{1}$-ATPase $\alpha$-subunit was detected in the acid-sensitive mutant. After BLAST analysis of the DGCC $12411^{\mathrm{m}}$ ATPase gene against the whole-genome sequences of other L. plantarum strains in GenBank, we found that this region is extremely conserved (Figure 3 ). In a previous study, the mutations in 3 LPA L. plantarum mutants that were generated using a similar method occurred on the $\mathrm{F}_{0} \mathrm{~F}_{1}$-ATPase $\beta$-subunit (Jia et al., 2013). Jia et al. (2013) also reported that mutations that occurred in or around the subunit-subunit interaction interface resulted in a pro- found reduction of ATPase activity, but mutations that took place outside or far from the motif did not show this effect. Jaichumjai et al. (2010) have also reported on the generation of acid-sensitive L. plantarum strains, but the mutations were not identified in their study. In DGCC $12411^{\mathrm{m}}$, the mutation from $\mathrm{G}$ to $\mathrm{A}$ at position 506 resulted in a change from a glycine residue at position 169 to an aspartic acid residue. Glycine-169 is located as the first glycine residue at the Walker A motif (also known as a phosphate-binding loop or a P-loop), a glycine-rich loop that commonly presents in the $\alpha$ and $\beta$-subunits of $\mathrm{F}_{1}$-ATPase, myosin, and other ATPutilizing enzymes (Walker et al., 1982; Ramakrishnan et al., 2002). The P-loop consists of a sequence of 8 amino acids [GXXXXGKT(S), G: glycine; K: lysine; T: threonine; S: serine; X: any amino acid], with the first glycine residue highly conserved in enzymes containing this motif across archaea, prokaryotes, and eukaryotes (Kumar et al., 2010), and critical to the enzymatic activity. For example, Priya et al. (2011) demonstrated that the substitution of the first glycine residue with alanine in the catalytic A-subunit of A-ATPase of Pyrococcus horikoshii OT3 resulted in flattening of the P-loop conformation, which inevitably closed the active site region and hindered nucleotide binding.

\section{Stability Assessment of LPA Mutant DGCC12411 ${ }^{m}$}

Due to the different growth environments and selective pressures between laboratory experiments and commercial-scale production, phenotype stability is one of the major concerns in the application of mutants in industrial production, especially with mutations in the enzymes that are core to bacterial activity. Despite $\mathrm{F}_{0} \mathrm{~F}_{1}$-ATPase-based LPA LAB mutants being developed and reported in several previous studies (Yamamoto et al., 1996; Jaichumjai et al., 2010; Jia et al., 2013), their stability has rarely been assessed. A previous study reported on the stability of an uncharacterized acidsensitive L. delbrueckii ssp. bulgaricus mutant (Möller et al., 2007). During continuous passage in milk, they observed a continuous reduction in the final $\mathrm{pH}$ by the mutant, without characterizing the genetic changes that contributed to the LPA phenotype nor the cause of the phenotype instability.

In this study, we found that the back mutation from aspartic acid residue to glycine reverses the postfermentation acidification phenotype. Therefore, we believe the single amino acid residue change at this conserved motif of $\mathrm{F}_{0} \mathrm{~F}_{1}$-ATPase $\alpha$-subunit caused the LPA phenotype of DGCC $12411^{\mathrm{m}}$. To verify our postulation, the stability of the SNP mutation as well as the LPA phenotype of DGCC $12411^{\mathrm{m}}$ were assessed across 10 passages [estimated 33 generations, 10\% (vol/vol) 


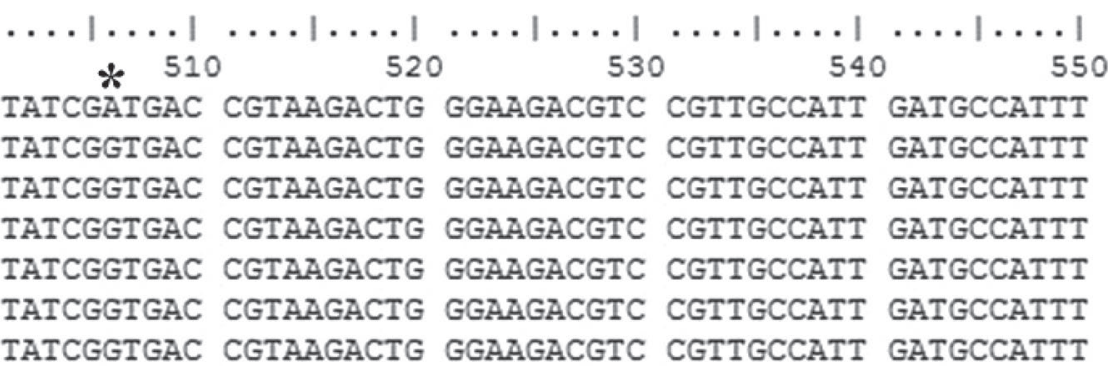

Figure 3. Alignment of the complete gene sequences of $\mathrm{F}_{0} \mathrm{~F}_{1}$-ATPase $\alpha$-subunit of Lactobacillus plantarum DGCC12411 and its mutant strain, DGCC12411 ${ }^{\mathrm{m}}$, with other L. plantarum strains deposited in the National Center for Biotechnology Information (NCBI, Bethesda, MD) with 99.87\% homology: L. plantarum WCFS1 (NC_004567.2), L. plantarum DR7 (CP031318.1), L. plantarum JDM1 (CP001617.1), L. plantarum P-8 (CP005942.2), and L. plantarum ST-III (CP002222.1). Site of SNP mutation (position 506) marked with an asterisk (*).

inoculation], which is about $50 \%$ more than standard industrial practice (about 22 generations). Figure 4A shows that the $\mathrm{pH}_{48 \mathrm{~h}}$ of DGCC12411 ${ }^{\mathrm{m}}$ decreased gradually from $\mathrm{pH} 4.15 \pm 0.00$ at $\mathrm{P} 1$ to $3.94 \pm 0.05$ at $\mathrm{P} 5$ (about 17 generations) and drastically dropped to 3.77 $\pm 0.03(\mathrm{SD})$ at $\mathrm{P} 6$ (about 20 generations), which was similar to results for the mother strain, DGCC12411. Then, the $\mathrm{pH}_{48 \mathrm{~h}}$ remained stable until P10. Such low stability in a phenotype could cause problems during strain preservation and production. To further understand the post-fermentation acidification evolution in this population, 30 single colonies were isolated from each passage, and their post-fermentation acidification phenotype was determined. Figure 5 shows that the $\mathrm{pH}_{48 \mathrm{~h}}$ of all colonies from $\mathrm{P} 1$ to $\mathrm{P} 4$ remained stable and was not significantly different than that of DGCC12411 ${ }^{\mathrm{m}}$. At P5 (about 17 generations), variants emerged with significantly $(P<0.01)$ lower end $\mathrm{pH}$ $\left(\mathrm{pH}_{48 \mathrm{~h}}<4.0\right)$, which accounted for $43 \%$ (13 out of 30) of the colonies. These low- $\mathrm{pH}_{48 \mathrm{~h}}$ colonies became dominant from P6 to P10 (around 20 to 33 generations) in all 3 replicates. This result showed a clear evolution of the dominant population from LPA to a normal postfermentation acidification phenotype.

It is known that during adaptation to stressful conditions, genetic changes might occur to restore cellular physiology to its original state (Carroll and Marx 2013), especially when such restorations possess a selective advantage or favor the survival of the cells (Wright and Minnick, 1997). Phenotype restorations have been observed during metabolic perturbation and high-temperature adaptation (Fong et al., 2005; Carroll and Marx 2013; Hug and Gaut, 2015). These revertants might be due to the compensatory changes from one or more mutations in a different gene or genes, one or more regulatory signals within the original gene but at a site other than the causal mutation, or a true reversion that restored the original amino acid sequence (Holmes and Jobling, 1996; Wright and Minnick, 1997).
To understand whether the LPA phenotype degeneration was caused by back mutation of GAT to GGT, the $\mathrm{F}_{0} \mathrm{~F}_{1}$-ATPase $\alpha$-subunit genes of the population (pooled from 3 replicates) from each passage were analyzed by Sanger sequencing. We noticed that an SNP back mutation appeared at P5 (about 17 generations) and became dominant after P7 (about 23 generations; Figure 4B). To further understand whether the SNP back mutation is the only cause of the LPA phenotype degeneration, the $\mathrm{F}_{0} \mathrm{~F}_{1}$-ATPase $\alpha$-subunit of these 300 isolates were sequenced (Figure 5). All the colonies isolated from P1 to $\mathrm{P} 4$ (up to 13 generations) were GAT at positions 505 to 507. In P5 (about 17 generations), 3 GGT colonies were detected, which accounted for $10 \%$ of the isolates. These GGT colonies indicated that a true reversion occurred and restored the original amino acid sequence. The proportion of GGT colonies increased to $100 \%$ after P6 (about 20 generations) in replicate 3 (Figure $5 \mathrm{C})$. However, we also noticed that not all low- $\mathrm{pH}_{48 \mathrm{~h}}$ colonies were GGT. The GGT colonies accounted for only $48.7 \%$ (79 out of 162 ) of the low- $\mathrm{pH}_{48 \mathrm{~h}}$ colonies. These GAT colonies with low $\mathrm{pH}_{48 \mathrm{~h}}$ suggested that, beyond true reversion, other mechanisms were involved that compensated for its acid tolerance. Möller et al. (2007) have reported that a continuous decrease in the $\mathrm{pH}$ of variants isolated from each passage was caused by a phenotypic adaptation rather than by genetic reversion. However, other enzymes, such as glutamate decarboxylase and arginine deiminase, also play important roles in pH homeostasis (Jaichumjai et al., 2010; Huang et al., 2016; Wu et al., 2017). To understand the cause of GAT colonies with low $\mathrm{pH}_{48 \mathrm{~h}}$, their wholegenome sequences will be analyzed in the future.

\section{Improvement of the LPA Phenotype Stability}

Inoculation size affected the initial population of bacteria in the fermentation and is a parameter critical to bacterial growth and acid production (Stevens et al., 


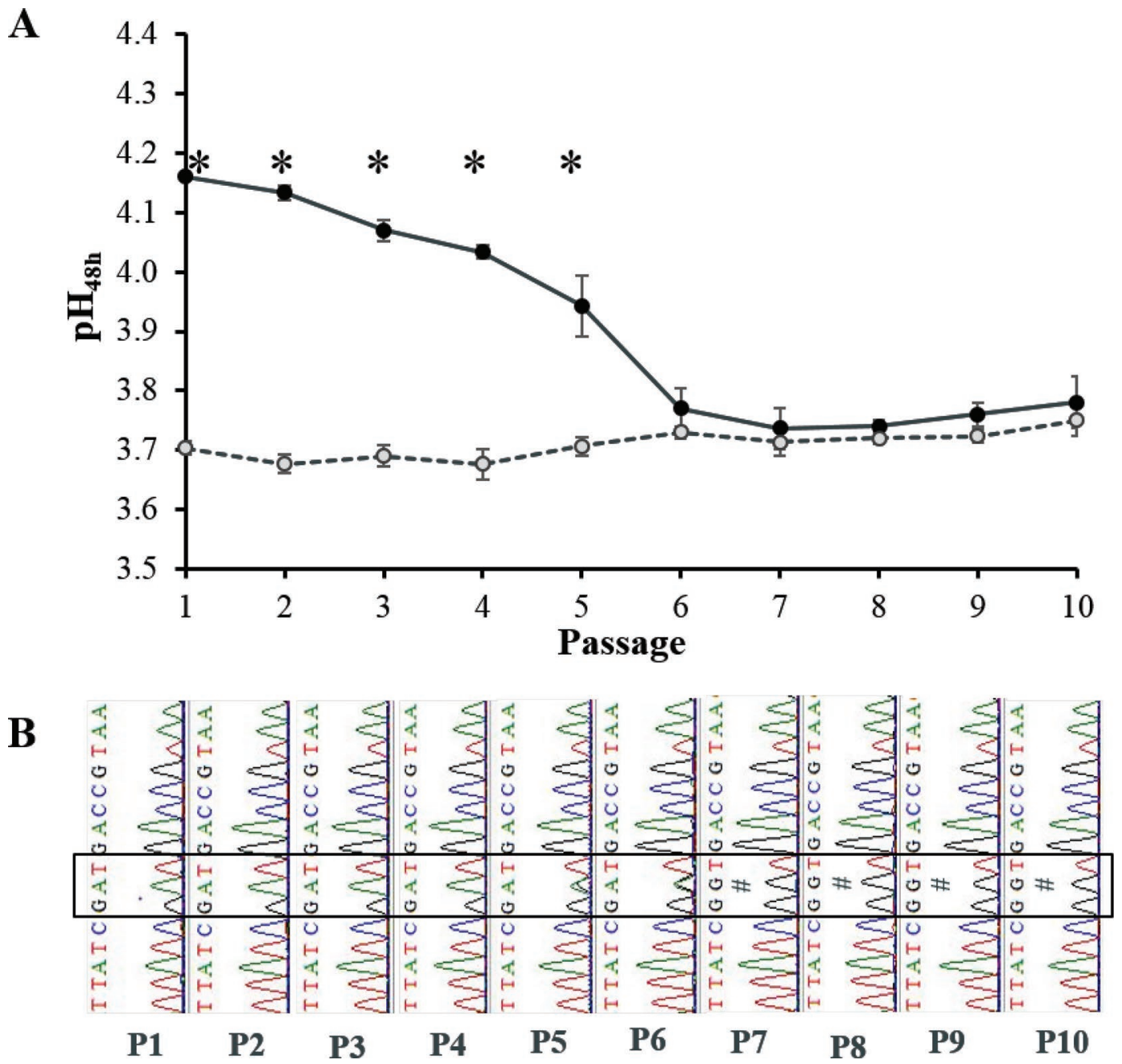

Figure 4. Stability of the low post-fermentation acidification phenotype and genotype during passaging. (A) pH of the 48-h culture of acidsensitive mutant Lactobacillus plantarum DGCC12411 ${ }^{\mathrm{m}}(\bullet$, solid line) and L. plantarum DGCC12411 (O, dashed line) in fresh De Man, Rogosa, and Sharpe medium for 10 passages. Asterisks $\left(^{*}\right)$ denote significant differences $(P<0.01)$ between $\mathrm{pH}$ of DGCC12411 ${ }^{\mathrm{m}}$ and its mother strain at different passages. Inoculation rate was 10\% (vol/vol). Error bars represent SD of 3 replicates. (B) Alignment of the partial gene sequences of $\mathrm{F}_{0} \mathrm{~F}_{1}$-ATPase $\alpha$-subunit of DGCC $12411^{\mathrm{m}}$ broth culture (pooled from 3 replicates) from P1 to P10. Site of SNP mutation (position 506) marked with \#; the 3 codons inside the frame encoded for glycine-aspartic acid-169.

2008; Wardani et al., 2017). With a higher inoculation level, the probability of transferring the revertant to the next passage will increase. In addition, it will also shorten the time to stationary phase, resulting in a longer exposure time at lower $\mathrm{pH}$ for bacteria cultivated for $24 \mathrm{~h}$. To assess the effects of inoculation ratio on end $\mathrm{pH}$ during passaging, we compared the $\mathrm{pH}_{48 \mathrm{~h}}$ of DGCC $12411^{\mathrm{m}}$ with $10 \%$ and $2 \%$ inoculum from $\mathrm{P} 1$ to P10; these inocula are estimated to grow about 3.3 and 5.6 generations per passage, respectively. We used MRS supplemented with neomycin sulfate $(600 \mu \mathrm{g} / \mathrm{mL})$ as a reference. As expected, with the strong selective pressure of neomycin, the LPA phenotype of DGCC $12411^{\mathrm{m}}$ was extended up to P9. Compared with the $10 \%$ inoculation rate, for which $\mathrm{pH}_{48 \mathrm{~h}}$ started to drop after
$\mathrm{P} 4$ (about 13 generations), the $\mathrm{pH}_{48 \mathrm{~h}}$ of $\mathrm{DGCC} 12411^{\mathrm{m}}$ inoculated at $2 \%$ was significantly $(P<0.05)$ higher from P5 to P7 (28 to 40 generations), which demonstrated an improvement of LPA stability (Figure 6A).

In addition, prolonged acid stress exposure is generally more favorable for the selection of variants that can grow to a lower $\mathrm{pH}$, which may be adverse for the maintenance of the LPA phenotype. Therefore, we speculated that relief of $\mathrm{H}^{+}$stress during $\mathrm{pH}$ homeostasis by addition of buffering salt in the growth medium may help extend the stability. Previous study has also reported on supplementing buffering salts (such as potassium phosphate) as a $\mathrm{pH}$ stabilizer during fermentation (Lee et al., 2009). Adding $0.05 \mathrm{M}$ potassium phosphate buffer into the medium slightly improved 
$\mathbf{A}$

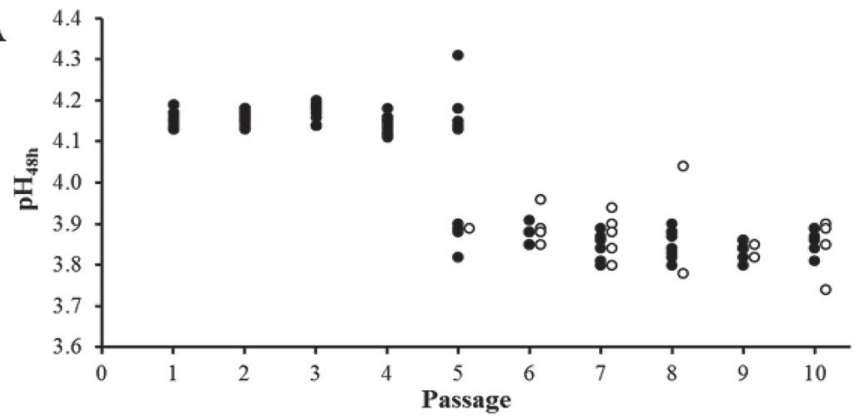

B
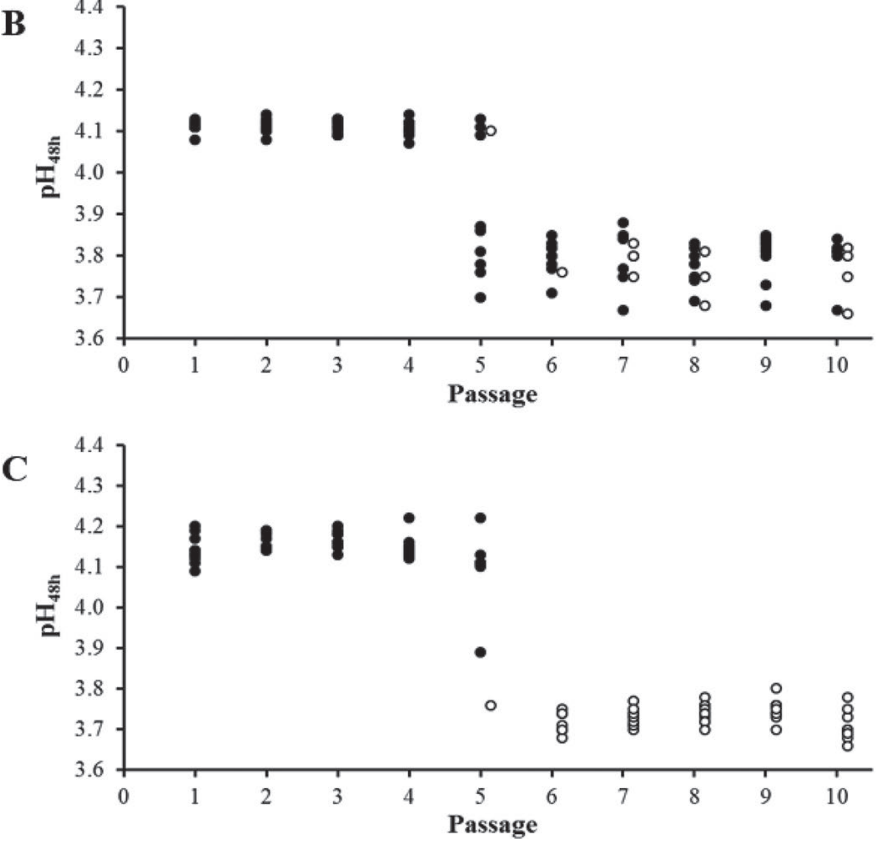

Figure 5. Stability of the low post-fermentation acidification phenotypes of the acid-sensitive mutant Lactobacillus plantarum DGCC $12411^{\mathrm{m}}$ over 10 passages. $\mathrm{A}=$ replicate $1 ; \mathrm{B}=$ replicate $2 ; \mathrm{C}=$ replicate 3. represents colonies with GAT at positions 505 to 507; $\bigcirc$ represents colonies with GGT at positions 505 to 507 . Inoculation rate was $10 \%$ ( $\mathrm{vol} / \mathrm{vol}) \cdot \mathrm{pH}_{48 \mathrm{~h}}=\mathrm{pH}$ after $48 \mathrm{~h}$ of cultivation.

the $\mathrm{pH}_{48 \mathrm{~h}}$ stability of DGCC12411 ${ }^{\mathrm{m}}$ (Figure 6B). Greater improvement in the stability of DGCC12411 ${ }^{\mathrm{m}}$ was observed when we supplemented $0.1 \mathrm{M}$ potassium phosphate buffer to MRS, even though significant $(P$ $<0.05)$ improvement compared with the untreated control was only observed at $\mathrm{P} 10$. The $\mathrm{pH}_{48 \mathrm{~h}}$ stability of DGCC12411 ${ }^{\mathrm{m}}$ can be maintained at the same level up to P8 (about 45 generations), which is twice the number of generations for strain scale-up in industrial production practices.

\section{CONCLUSIONS}

In this study, an acid-sensitive L. plantarum mutant was developed, which showed improved post-fermentation acidification under laboratory conditions, as well
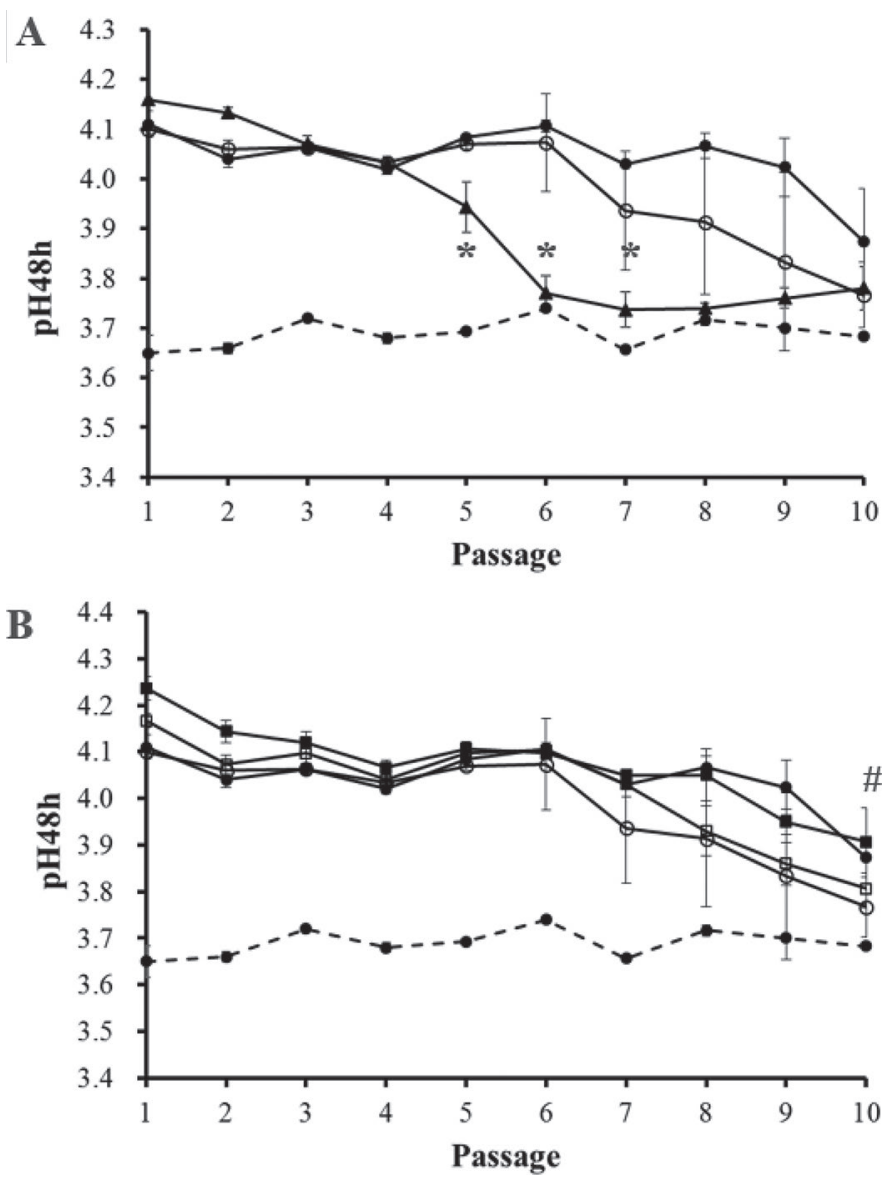

Figure 6. Effects of (A) inoculation size and (B) potassium phosphate buffer on the stability of the low post-fermentation acidification phenotype (as measured by final $\mathrm{pH}$ at $48 \mathrm{~h}$ ) of the acid-sensitive mutant of Lactobacillus plantarum DGCC12411, designated as DGCC $12411^{\mathrm{m}}$. $($ solid line $)=$ DGCC $12411^{\mathrm{m}}$ in De Man, Rogosa, and Sharpe medium (MRS) supplemented with neomycin sulfate $(600 \mu \mathrm{g} /$ $\mathrm{mL}$, positive control); $\bullet$ (dashed line) = DGCC12411 in MRS (negative control); $\boldsymbol{\Delta}=10 \%(\mathrm{vol} / \mathrm{vol})$ DGCC $12411^{\mathrm{m}}$ in MRS; $\bigcirc=2 \%$ (vol/vol) DGCC12411 ${ }^{\mathrm{m}}$ in MRS (untreated control for panel B); $=$ DGCC $12411^{\mathrm{m}}$ in MRS supplemented with $0.1 \mathrm{M}$ potassium phosphate buffer; $\square=$ DGCC12411 ${ }^{\mathrm{m}}$ in MRS supplemented with $0.05 \mathrm{M}$ potassium phosphate buffer. Error bars represent SD of 3 replicates. Asterisks $\left(^{*}\right)$ denote significant differences $(P<0.05)$ in $\mathrm{pH}$ between $10 \%(\mathrm{vol} / \mathrm{vol})$ and $2 \%(\mathrm{vol} / \mathrm{vol})$ DGCC12411 ${ }^{\mathrm{m}}$ in MRS. Hash sign (\#) denotes significant difference $(P<0.05)$ in $\mathrm{pH}$ between $0.1 M$ potassium phosphate buffer and untreated control.

as improved shelf-life $\mathrm{pH}$ stability in the ambient yogurt application model for up to 4 mo. We identified that a novel SNP mutation (which resulted in the replacement of a highly conserved glycine residue by aspartic acid) in the $\mathrm{F}_{0} \mathrm{~F}_{1}$-ATPase $\alpha$-subunit led to the LPA phenotype. We also demonstrated that the mutant developed in the laboratory using strong selective pressure could have issues in phenotype and genotype stability during industrial-scale production, as back mutation occurred after 5 passages (about 17 generations). Stabilizing the mutation is of utmost importance if the strain is to be 
used for future industry application, as degeneration of the LPA phenotype will negatively affect the benefits of the desired phenotype in commercial fermented dairy products, especially those stored at ambient temperature. In view of this, a thorough assessment of genotype and phenotype stability is of utmost importance during strain functionality improvement. Moreover, we also developed methods to stabilize the LPA phenotype during passaging, which may enable the industrial application of such mutants. This study provided a practical way for developing milder starter cultures for industrial application.

\section{ACKNOWLEDGMENTS}

This work was funded by DuPont Nutrition and Biosciences, Shanghai, China. The authors thank Julia Salabai (intern from Erhvervsakademi Aarhus, Aarhus, Denmark) and Meng Chen (technician, DuPont $\mathrm{Nu}$ trition and Biosciences) for their contribution to the experiments, and Dennis Romero (technical fellow, DuPont Nutrition and Biosciences) and Cindy Stewart (Global Technology and Innovation Leader, DuPont Nutrition and Biosciences) for their support and comments on the manuscript. The authors have not stated any conflicts of interest.

\section{REFERENCES}

Bernardeau, M., M. Guguen, and J. P. Vernoux. 2006. Beneficial lactobacilli in food and feed: Long-term use, biodiversity and proposals for specific and realistic safety assessments. FEMS Microbiol. Rev. 30:487-513. https://doi.org/10.1111/j.1574-6976.2006.00020.x.

Carroll, S. M., and C. J. Marx. 2013. Evolution after introduction of a novel metabolic pathway consistently leads to restoration of wild-type physiology. PLoS Genet. 9:e1003427. https://doi.org/10 .1371/journal.pgen.1003427.

Chen, S., L. Chen, L. Chen, X. Ren, H. Ge, B. Li, G. Ma, X. Ke, J. Zhu, L. Li, Y. Feng, and Y. Li. 2018. Potential probiotic characterization of Lactobacillus reuteri from traditional Chinese highland barley wine and application for room-temperature-storage drinkable yogurt. J. Dairy Sci. 101:5780-5788. https://doi.org/10.3168/ jds.2017-14139.

Cotter, P. D., and C. Hill. 2003. Surviving the acid test: Responses of gram-positive bacteria to low $\mathrm{pH}$. Microbiol. Mol. Biol. Rev. 67:429-453. https://doi.org/10.1128/MMBR.67.3.429-453.2003.

de J. C. Munanga, B., G. Loiseau, J. Grabulos, and C. Mestres. 2016. Modeling lactic fermentation of Gowé using Lactobacillus starter culture. Microorganisms 4:44. https://doi.org/10.3390/ microorganisms4040044.

Fong, S. S., A. R. Joyce, and B. Ø. Palsson. 2005. Parallel adaptive evolution cultures of Escherichia coli lead to convergent growth phenotypes with different gene expression states. Genome Res. 15:1365-1372. https://doi.org/10.1101/gr.3832305.

Freire, A. L., C. L. Ramos, P. N. da Costa Souza, M. G. B. Cardoso, and R. F. Schwan. 2017. Nondairy beverage produced by controlled fermentation with potential probiotic starter cultures of lactic acid bacteria and yeast. Int. J. Food Microbiol. 248:39-46. https://doi.org/10.1016/j.ijfoodmicro.2017.02.011.

Gomez, S., S. Rebiffe, P. Horvath, C. Fremaux, and P. Fourcassie. 2017. Streptococcus thermophilus strain exhibiting unusual acidifi- cation kinetics in milk. P. 106 in 12th International Symposium of Lactic Acid Bacteria Abstract Book. Egmond aan Zee, the Netherlands.

Holmes, R. K., and M. G. Jobling. 1996. Chapter 5: Genetics. In Medical Microbiology. 4th ed. S. Baron S, ed. University of Texas Medical, Galveston, TX. https://www.ncbi.nlm.nih.gov/books/ NBK7908/.

Huang, R., M. Pan, C. Wan, N. P. Shah, X. Tao, and H. Wei. 2016. Physiological and transcriptional responses and cross protection of Lactobacillus plantarum ZDY2013 under acid stress. J. Dairy Sci. 99:1002-1010. https://doi.org/10.3168/jds.2015-9993.

Hug, S. M., and B. S. Gaut. 2015. The phenotypic signature of adaptation to thermal stress in Escherichia coli. BMC Evol. Biol. 15:177-188. https://doi.org/10.1186/s12862-015-0457-3.

Jaichumjai, P., R. Valyasevi, A. Assavanig, and P. Kurdi. 2010. Isolation and characterization of acid-sensitive Lactobacillus plantarum with application as starter culture for Nham production. Food Microbiol. 27:741-748. https://doi.org/10.1016/j.fm.2010.03.014.

Jia, B. X., X. Zhong, C. Yuan, K. Li, K. Lin, Q. H. Zhang, Z. Che, G. Chen, and W. Xiang. 2013. Screening of Lactobacillus plantarum LPM21 with $\mathrm{F}_{0} \mathrm{~F}_{1}$-ATPase $\beta$-subunit mutation used as probiotics adjunct in Sichuan pickle. Food Sci. Technol. Res. 19:1045-1050. https://doi.org/10.3136/fstr.19.1045.

Kanner, B. I., and D. L. Gutnick. 1972. Use of neomycin in the isolation of mutants blocked energy conservation in Escherichia coli. J. Bacteriol. 111:287-289. https://doi.org/10.1128/JB.111.1.287-289 .1972.

Koumandou, V. L., and S. Kossida. 2014. Evolution of the $\mathrm{F}_{0} \mathrm{~F}_{1}$ ATP synthase complex in light of the patchy distribution of different bioenergetic pathways across prokaryotes. PLOS Comput. Biol. 10:e1003821. https://doi.org/10.1371/journal.pcbi.1003821.

Kumar, A., M. S. S. Manimekalai, A. M. Balakrishna, J. Jeyakanthan, and G. Grüber. 2010. Nucleotide-binding states of subunit A of the A-ATP synthase and the implication of P-loop switch in evolution. J. Mol. Biol. 396:301-320. https://doi.org/10.1016/j.jmb .2009.11.046.

Kurdi, P., T. Smitinont, and R. Valyasevi. 2009. Isolation and characterization of acid-sensitive mutants of Pediococcus acidilactici. Food Microbiol. 26:82-87. https://doi.org/10.1016/j.fm.2008.08 .006 .

Lee, N.-K., Y.-B. Jo, I. H. Jin, C.-W. Son, and J.-W. Lee. 2009. The effect of potassium phosphate as a $\mathrm{pH}$ stabilizer on the production of gellan by Sphingomonas paucibilis NK-2000. J. Life Sci. 19:1033-1038. https://doi.org/10.5352/JLS.2009.19.8.1033.

Liu, L., C. Li, and J. Liu. 2017. Rheological and physical characteristics of non-fat set yogurt prepared with EPS-producing Streptococcus thermophilus and an $\mathrm{H}^{+}$-ATPase-defective mutant Lactobacillus delbrueckii ssp. bulgaricus. Int. J. Food Prop. 20:745-753. https://doi.org/10.1080/10942912.2016.1180531.

Mao, Y.J. 2019. New Lactobacillus plantarum strain and uses thereof. World Intellectual Property Organization (WO2019061263A1).

Möller, C., W. Bockelmann, A. Ammann, and K. J. Heller. 2007. Production of yoghurt with mild taste by a Lactobacillus delbrueckii ssp. bulgaricus mutant with altered proteolytic properties. Biotechnol. J. 2:469-479. https://doi.org/10.1002/biot.200600225.

Morgulis, A., G. Coulouris, Y. Raytselis, T. L. Madden, R. Agarwala, and A. A. Schäffer. 2008. Database indexing for production MegaBLAST searches. Bioinformatics 24:1757-1764. https://doi.org/10 .1093/bioinformatics/btn322.

Nannen, N. L., and R. W. Hutkins. 1991. Proton-translocating adenosine triphosphatase activity in lactic acid bacteria. J. Dairy Sci. 74:747-751. https://doi.org/10.3168/jds.S0022-0302(91)78220-9.

Nedelcheva, P., Z. Denkova, P. Denev, A. Slavchev, and A. Krastanov. 2010. Probiotic strain Lactobacillus plantarum NBIMCC 2415 with antioxidant activity as a starter culture in the production of dried fermented meat products. Biotechnol. Biotechnol. Equip. 24:16241630. https://doi.org/10.2478/V10133-010-0016-4.

Ongol, M. P., Y. Sawatari, Y. Ebina, T. Sone, M. Tanaka, F. Tomita, A. Yokota, and K. Asano. 2007. Yoghurt fermented by Lactobacillus delbrueckii ssp. bulgaricus $\mathrm{H}^{+}$-ATPase-defective mutants exhibits enhanced viability of Bifidobacterium breve during storage. 
Int. J. Food Microbiol. 116:358-366. https://doi.org/10.1016/j .ijfoodmicro.2007.02.019.

Piard, J. C., and M. Desmazeaud. 1991. Inhibiting factors produced by lactic acid bacteria. 1 . Oxygen metabolites and catabolism endproducts. Lait 71:525-541. https://doi.org/10.1051/lait:1991541.

Priya, R., A. Kumar, M. S. Manimekalai, and G. Grüber. 2011. Conserved glycine residues in the P-loop of ATP synthases form a doorframe for nucleotide entrance. J. Mol. Biol. 413:657-666. https://doi.org/10.1016/j.jmb.2011.08.045.

Ramakrishnan, C., V. S. Dani, and T. Ramasarma. 2002. A conformational analysis of Walker motif A [GXXXXGKT (S)] in nucleotidebinding and other proteins. Protein Eng. 15:783-798. https://doi .org/10.1093/protein/15.10.783.

Stevens, M. J. A., A. Wiersma, W. M. de Vos, O. P. Kuipers, E. J. Smid, D. Molenaar, and M. Kleerebezem. 2008. Improvement of Lactobacillus plantarum aerobic growth as directed by comprehensive transcriptome analysis. Appl. Environ. Microbiol. 74:47764778. https://doi.org/10.1128/AEM.00136-08.

Tamang, J. P., K. Watanabe, and W. H. Holzapfel. 2016. Review: Diversity of microorganisms in global fermented foods and beverages. Front. Microbiol. 7:377. https://doi.org/10.3389/fmicb.2016 .00377 .

Walker, J. E., M. Saraste, M. J. Runswick, and N. J. Gay. 1982. Distantly related sequences in the alpha- and beta-subunits of ATP synthase, myosin, kinases and other ATP-requiring enzymes and a common nucleotide binding fold. EMBO J. 1:945-951. https://doi .org/10.1002/j.1460-2075.1982.tb01276.x.

Wardani, S. K., M. N. Cahyanto, E. S. Rahayu, and T. Utami. 2017. The effect of inoculum size and incubation temperature on cell growth, acid production and curd formation during milk fermentation by Lactobacillus plantarum Dad 13. Int. Food Res. J. 24:921926.

Wright, B. E., and M. F. Minnick. 1997. Reversion rates in a leuB auxotroph of Escherichia coli K-12 correlate with ppGpp levels during exponential growth. Microbiology 143:847-854. https://doi .org/10.1099/00221287-143-3-847.

Wu, Q., H. M. Tun, Y. S. Law, E. Khafipour, and N. P. Shah. 2017. Common distribution of gad operon in Lactobacillus brevis and its GadA contributes to efficient GABA synthesis toward cytosolic near-neutral pH. Front. Microbiol. 8:206. https://doi.org/10.3389/ fmicb.2017.00206.

Yamamoto, N., Y. Masujima, and T. Takano. 1996. Reduction of membrane-bound ATPase activity in a Lactobacillus helveticus strain with slower growth at low $\mathrm{pH}$. FEMS Microbiol. Lett. 138:179-184. https://doi.org/10.1111/j.1574-6968.1996.tb08153.x.

Yang, J., Y. Cao, Y. Cai, and F. Terada. 2010. Natural populations of lactic acid bacteria isolated from vegetable residues and silage fermentation. J. Dairy Sci. 93:3136-3145. https://doi.org/10.3168/ jds.2009-2898.

Yokota, A., S. Amachi, S. Ishii, and F. Tomita. 1995. Acid sensitivity of a mutant of Lactococcus lactis ssp. lactis $\mathrm{C} 2$ with reduced membrane-bound ATPase activity. Biosci. Biotechnol. Biochem. 59:2004-2007. https://doi.org/10.1271/bbb.59.2004.

Yu, J., H. M. Wang, M. S. Zha, Y. T. Qing, N. Bai, Y. Ren, X. X. Xi, W. J. Liu, B. L. G. Menghe, and H. P. Zhang. 2015. Molecular identification and quantification of lactic acid bacteria in traditional fermented dairy foods of Russia. J. Dairy Sci. 98:5143-5154. https://doi.org/10.3168/jds.2015-9460.

Zhang, Z., S. Schwartz, L. Wagner, and W. Miller. 2000. A greedy algorithm for aligning DNA sequences. J. Comput. Biol. 7:203-214. https://doi.org/10.1089/10665270050081478.

\section{ORCIDS}

L.-O. Chuah @ https://orcid.org/0000-0003-4740-8620

Y. Mao @ https://orcid.org/0000-0001-9036-8770 